ÉGYPTE

monde arabe

\section{Égypte/Monde arabe}

32 | 1997

Mélanges

\title{
Libéralité prétorienne et État minimum au Soudan
}

L'effort civique entre la poudre et les travaux publics

\section{Isabelle Baillard et Patrick Haenni}

\section{(2) OpenEdition}

Journals

Édition électronique

URL : https://journals.openedition.org/ema/1671

DOI : 10.4000/ema.1671

ISSN : 2090-7273

Éditeur

CEDEJ - Centre d'études et de documentation économiques juridiques et sociales

Édition imprimée

Date de publication : 31 décembre 1997

Pagination : 71-96

ISSN : 1110-5097

Référence électronique

Isabelle Baillard et Patrick Haenni, «Libéralité prétorienne et État minimum au Soudan », Égypte/ Monde arabe [En ligne], 32 | 1997, mis en ligne le 08 juillet 2008, consulté le 07 juillet 2022. URL : http:// journals.openedition.org/ema/1671; DOI : https://doi.org/10.4000/ema.1671

Ce document a été généré automatiquement le 7 juillet 2022

Tous droits réservés 


\section{Libéralité prétorienne et État minimum au Soudan}

L'effort civique entre la poudre et les travaux publics

Isabelle Baillard et Patrick Haenni

"OOn ne commence à parler d'État que lorsque
des hommes ou des organismes qui assurent
plusieurs services publics commencent à être
réunis entre eux et par là à faire masse. ${ }^{\circ}$ "
Paul Veyne, Le pain et le cirque. Sociologie d'un
pluralisme politique.

$1 \mathrm{Au}$ Soudan, comme ailleurs, l'édification de l'espace public ${ }^{1}$ n'a suivi que marginalement la voie royale de l'administration exclusive par l'État. S'appuyant largement sur la philanthropie et le civisme de ses gouvernés, le régime islamique, comme ses prédécesseurs, a révélé son incapacité ou son indifférence vis-à-vis d'une intégration complète de l'administration de la sphère publique dans le champ étatique. Dès lors, l'espace public en constitution devenait terre d'alliances et d'échanges incessants entre les élites au pouvoir et les acteurs de la société civile. Grands marchands en tête, ces derniers se sont investis et s'investissent encore, dans l'édification du paysage urbain et l'amélioration du service public - en sachant monnayer, souvent au prix fort, les contreparties de leur engagement civique. Avant et pendant la colonisation britannique, et sans originalité aucune, l'évergétisme social ${ }^{2}$, étayé par les répertoires islamiques d'une générosité redistributive ${ }^{3}$, a été l'un des moyens de prédilection mobilisé par les grands marchands soudanais afin de s'attirer les faveurs du pouvoir et de promouvoir ou consolider ainsi leurs intérêts économiques.

2 On peut suivre sans risque la sagesse du peuple - qui qualifie volontiers ces donations de " $^{\circ}$ charités à contrepartie ${ }^{\circ} »(\text { khayr bi muqâbil })^{4}$ - et douter avec lui de la pureté des intentions des donateurs. L'essentiel toutefois est ailleurs et réside dans ce constat que l'espace public a dû et doit encore beaucoup à l'évergétisme. Les notables urbains ( $a$ 'yân al-madîna), ont parsemé le paysage des cités de leurs œuvres, les pourvoyant généreusement en écoles, jardins et hôpitaux. Ils ont élevé des mosquées, installé des 
égouts ou des chaussées. Scénario banal des États émergents ${ }^{5}$, la production de l'espace public au Soudan fait jusqu'aujourd'hui la part belle au dhât, un champ de pratiques dont l'évergétisme n'est qu'une partie. La notion anglaise de self rend assez fidèlement le sens du terme - mieux en tous cas que le " $^{\circ}$ soi ${ }^{\circ}$ » français - dans ses différentes variantes de 'awn dhâtî (self-help), i'timâd 'ala-l-dhât (self reliance), juhûd dhâtiyya (self efforts) ${ }^{6}$. En termes génériques, le qualificatif dhât s'étend donc au-delà des œuvres pour inclure toute action qui procède de ce que Nadia Khouri-Dagher désigne ailleurs par " ${ }^{\circ}$ answer to a defaulting state ${ }^{\circ}$ " (1987). Il comprend toutes les initiatives lancées en faveur du bien commun qui sont en position d'extériorité proclamée par rapport à la sphère de l'État. Mais proclamation n'est pas raison': l'empressement avec lequel les têtes pensantes du régime actuel invoquent et saluent les juhûd dhâtiyya - ces initiatives civiques dont l'existence est pourtant le symptôme le plus flagrant de l'échec de l'entreprise centralisatrice de l'État -, incite à penser que le champ de pratiques recouvert par le qualificatif dhât entretient avec l'État des rapports complexes qui ne sont pas purement de l'ordre de la compensation ou de l'évitement.

3 En cela, le sort réservé aux juhûd dhâtiyya constitue un analyseur privilégié pour explorer les logiques d'émergence du champ étatique ou, ce qui revient au même, les modalités du rapprochement organique entre la société civile et la société politique. L'inclination surprenante des élites dirigeantes à saluer les vertus du dhât nous permettra de relever la spécificité du rapport au champ social de l'État soudanaiso: alors que l'espace du dhât a été un peu partout dans le monde arabe - et l'on pense en particulier à l'Égypte et à l'Algérie - le site de formation et de localisation de contrepouvoirs aux appareils d'États, il a contribué au contraire au Soudan à précipiter l'annexion mutuelle des sociétés civile et politique.

Cet article explorera en premier lieu les modalités selon lesquelles les juhûd dhâtiyya sont passées d'une position d'altérité déclarée, par rapport au champ du pouvoir, à celle de pièce maîtresse et équivoque de la consolidation de l'État et de l'élite dirigeante. À ce propos, la tentation est grande d'adopter le point de vue de la

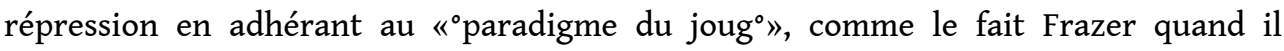
qualifie l'État actuel d'orwellian state (1995). Séduisante, la perspective est pourtant excessive $^{70}$ : ce qui se trame dans la fusion des sociétés politique et civile tient moins de l'absorption de la seconde par la première que de la mise en place d'un scénario intermédiaire " $^{\circ} \mathrm{d}$ 'annexion mutuelle ${ }^{\circ} »$. Ainsi, sous l'œil du pouvoir, les initiatives civiques, populaires ou édilitaires, ne cesseront de " ${ }^{\circ}$ réinventer de la différence ${ }^{\circ}$ » (Jean-François Bayart), de se défiler face à l'État, sans pour autant le défier.

Le rapport de ces initiatives à la guerre, des juhûd dhâtiyya aux juhûd harbiyya (l'effort de guerre), méritera ensuite notre attention puisqu'il met en question l'universalité des vertus pacifiques qu'Adam Smith attribuait au commerce. À ce titre, les libéralités explosives de tel grand marchand de Gedaref, ancien membre du Parti unioniste, devenu un célèbre avocat du jihad, opposent un démenti flagrant à l'angélisme de l'économiste écossais. Lorsque ce marchand décide en 1993, de sponsoriser une armée de $55^{\circ} 000$ personnes pour les Forces de défense populaire (quwwâtal-difâ'al-sha'bî), il fait contre bonne fortune bonne guerre ${ }^{\circ}$ : ce tribut prétorien lui vaut bien vite les faveurs du gouverneur de la ville qui l'invite sur le champ à rejoindre le Conseil municipal. Couvert d'honneurs, le nouveau conseiller municipal finit par décupler sa fortune en acquérant le plus grand hôtel de Gedaref, avant sa mort en 1996. Le commerce n'est pas $l^{\prime}$ antithèse de la guerre, pas plus d'ailleurs que le tribut ${ }^{80}$ : le " ${ }^{\circ}$ portefeuille charitable ${ }^{\circ}$ » 
des grands commerçants subit une inflexion notable dès la consolidation du régime islamiste. La redevance en espèces occupe une place toujours plus importante par rapport à l'évergétisme social, certains grands commerçants allant jusqu'à verser plus de $20^{\circ} \%$ de leurs revenus au Front national islamique (FNI) (Marchai, 1992). Parce que l'État est en mal de canons ${ }^{9}$, l'intrication du majhûd harbî et du majhûd dhâtî se resserre ${ }^{\text {: }}$ financer des armées en faveur du régime est devenu une opération certainement aussi avantageuse, sinon plus, en termes de reconnaissance politique, que l'érection de mosquées et d'hôpitaux ou que l'asphaltage des chaussées. Cette duplication des sites d'actualisation de la " $^{\circ}$ grandeur marchande ${ }^{\circ}$ " (participation à titre individuel à la chose publique $)^{10}$, tiraillés entre les œuvres et les libéralités en faveur de la guerre, n'est, au demeurant, que l'expression dans le monde marchand d'un phénomène recouvrant la société dans son intégralitéo: à savoir, l'imbrication des mécanismes de redistribution mis en place par la société civile (évergétisme social, mais aussi initiatives civiques des citoyens ordinaires) et des prélèvements informels en faveur de l'État et de la guerre (les tributs) - ou, comme beaucoup se plaisent à le dire, de " ${ }^{\circ} \mathrm{sa}^{\circ}$ » guerre.

Un deuxième thème se dessine ainsi au creux de ce basculement des initiatives citoyennes dans le champ d'attraction de l'État ${ }^{\circ}$ : l'intégration politique de la société. Un des avantages de l'expérience soudanaise est de couper court aux visions benoîtes et consensualistes des structuro-fonctionnalistes américains tant il ressort bien vite que l'intégration n'est pas la négation de la coercition. De deux façons, celle-ci participe en effet activement à la réalisation de celle-lào: en consolidant les technologies de contrôle social d'abord, et en accentuant l'extorsion rangée et policée des ressources de la société civile au profit du régime, ensuite. Cette " $^{\circ}$ mise en valeur de la sociétéo $»$ est organisée autour de trois visées contradictoires ${ }^{\circ}$ : la concentration de capital dans les mains de la classe dirigeante, le financement de la guerre et le développement des collectivités locales. L'incantation du régime au civisme citoyen (Le. aux juhûd dhâtiyya), cristallisée dans l'appel au i'timâd 'ala-l-dhât, ne doit pas faire illusion sur le contenu réel de la notion. En principe, l'expression renvoie bien à l'idée d'autogestion du local par lui-même, thème cher aux néolibéraux. Dans la pratique, il en va autrement ${ }^{\circ}$ : les modalités opératoires du i'timâd 'ala-l-dhât participent, de manière privilégiée, à la remise en ordre de la société par le régime.

\section{Les initiatives civiques ${ }^{\circ}$ : force de la société civile ou ruse de l'État minimum ${ }^{\circ}$ ?}

7 Khartoum 3, au sud-est de la capitale. L'espace public est saturé des réalisations d'un individu': Hassan 'Abd al-Mun'im, grand commerçant d'or des années quarante, dont la famille d'origine égyptienne s'est établie au Soudan au début du siècle dernier. Le jardin public, la place centrale, la mosquée, l'Institut d'études techniques, tous ont été financés par le pieux marchand. L'autre grand édifice évergétique de Khartoum 3, le Mujamma' Ibrâhîm Mâlik al-lslâmî (le Centre islamique Ibrâhîm Mâlik), est l'œuvre d'un des rares grands industriels du pays. Il a été édifié en 1984, peu après que l'ancien président Djafar al-Nimeiry eut décidé de conjuguer l'organisation de l'État et l'application de la sharia. Toujours sous le patronage d'Ibrâhîm Mâlik, un hôpital verra le jour, à côté de ce centre islamique qui abrite une mosquée, une école et une petite clinique. 
8 Foin des barrières temporelles pour ces édilités traditionnelles'! Elles ont traversé les changements de régimes et d'idéologies de ce siècle sans trop de heurts. N'est-ce pas au colonisateur anglais que Hassan 'Abd al-Mun'im remit la clé de son établissement scolaire afin qu'il soit administré par l'Occupant ${ }^{\circ}$ ? De son côté, Ibrâhîm Mâlik continua sous al-Nimeiry, nonobstant l'idéologie modernisatrice de l'Union socialiste, à reproduire cette vieille éthique marchande que constituent l'évergétisme et les œuvres. Cette dernière ne disparaîtra pas davantage après l'arrivée au pouvoir du gouvernement islamiste ${ }^{\circ}$ dans le quartier de Burrî, Wâd al-Jabal (pseudonyme de celui qui est vraisemblablement au Soudan le plus grand évergète actuel ${ }^{11}$ a érigé un complexe religieux, comprenant une école, une mosquée et un hôpital. Et il est loin de constituer une exception : à Hajj Yûsuf, sur l'autre bord du Nil, dans les nouveaux quartiers issus de l'exode des provinces, ce sont les notables locaux ou extérieurs, Soudanais d'autres régions du pays, hommes d'affaires du Golfe, ou encore, des individus proches des cercles du pouvoir, qui vont imprimer leur marque sur cette ébauche de paysage urbain. Alors que le ministre des Finances a versé 5 millions de livres soudanaises $(£ s)^{12}$ pour la construction de la municipalité, on trouve, un peu en retrait, l'école de la famille Alban Jadîd, la clinique al-Mayham al-Sharq de Abû alMa'âlî, le Centre de soins de Sayf Ibrâhîm Abdallah, les deux écoles de Sâdiq al-Hijâzî, ou encore l'École Sayf al-Harîr (le sabre de soie), financée par un entrepreneur koweïtien.

Ces évergésies constantes et inchangées dans leurs formes mises à part, la pratique du tribut a subi deux mutations au cours de ces dernières années.

En se militarisant tout d'abord, le tribut est devenu autrement plus contraignant. Au civisme séculaire des œuvres, devoir traditionnel du riche envers le pauvre ou l'État, est venue s'ajouter une nouvelle forme de participation informelle (Le., un versement en faveur de la collectivité, non médiatisé par la chambre de la zakât ou des impôts) de la classe marchande à la chose publique ${ }^{\circ}$ : la donation (tabarru), versée en liquide pour l'effort de guerre (majhûd harbi).

11 En se popularisant ensuite, et ce, contre la volonté du peuple, le tribut (complice, et non substitut de l'impôt), a fini par toucher des catégories de plus en plus nombreuses de la population. Aux a'mâl khayriyya, dont les juhûd dhâtiyya constituaient jusqu'alors la figure de proue, sont venues s'ajouter désormais les juhûd sha'biyya ou " ${ }^{\circ}$ efforts populaires ${ }^{\circ} »$. En effet, les individus ordinaires n'allaient pas demeurer en reste dans ces transformations de la contribution informelle à l'entreprise étatique. La vie associative de base, parent pauvre de l'édilité marchande, enregistra les mêmes mutations que celles subies par sa prestigieuse consœur. De part et d'autre, les espaces de la redistribution ou de la solidarité sociale se sont transformés en un levier implacable d'extraction des ressources de la société en faveur du système politique et militaire, et de ses leaders. Manière bien particulière d'assurer la pérennisation de l'État minimum au moindre coût, comme nous allons le voir.

12 En fait, il semble qu'en lieu et place d'un retrait ou d'un désengagement de l'État - et c'est en cela que le terme dhât s'avère illusoire - il y ait plutôt eu un redoublement de son ancrage institutionnel dans la société civile. Par le biais de politiques fiscales et redistributives, gérées par les Travaux publics et les Chambres des impôts et de la $z a k a t^{\circ}$; et par des échanges informels, tributs volontaires ou imposés, assortis ou non de contreparties, entre le réseau politique du gouvernement et son environnement social. 
13 Nous commencerons par un aperçu de la manière dont cette informatisation est inscrite dans une stratégie explicite du pouvoir. Nous reviendrons ensuite sur les rapports informels qu'entretiennent l'État et les marchands afin d'illustrer ce qui se joue dans cette informatisation du rapport de l'État à la société civile.

\section{Sites et procédures de l'annexion mutuelle}

\section{Le discours d'État et la doctrine de l'autosuffisance}

L'idée de juhûd dhâtiyya n'a pas attendu le régime islamiste pour voir le jour, l'État n'ayant jamais pu monopoliser le procès d'édification de l'espace public. Nombreux sont les bâtiments publics édifiés "०bil-'awn al-dhâtî̀"», grâce aux efforts civiques des collectivités locales (Abdou Maliqalim, 1994, chap. ${ }^{\circ 5}$ ). Le premier pas sur le chemin de l'annexion mutuelle du dhât et des stratégies urbaines de l'État a sans doute été l'établissement des cellules de base de l'Union socialiste en 1973 par Djafar al-Nimeiry. Entre autres tâches, il leur incombait d'organiser les initiatives populaires en matière de projets éducatifs ou de santé, assurant ainsi la distribution de services publics. En leur revenant de droit, l'administration du dhât devenait affaire d'État, d'autant que, pour la petite histoire, il leur fallait désormais recruter les $^{\circ}$ volontaires ${ }^{\circ} »$ pour aller grossir les rangs des ralliements de soutien au gouvernement. Le terme ne sera officialisé pour la première fois qu'en 1991. Il allait émerger à la faveur de deux enjeux ${ }^{\circ}$ : la détérioration du budget de l'État et l'exclusion du Soudan des allocations solidaires de la communauté internationale, et la hausse drastique de l'exode des provinces vers les quartiers périphériques de la capitale. Ces zones informelles ('ashwâ'iyyât) à l'avenir aléatoire jusqu'alors, promises à la destruction par les deux régimes précédents et maintenues sous pression policière permanente, allaient obtenir " $^{\circ}$ droit de cité ${ }^{\circ}$ ». En 1991, la nouvelle politique du régime, cristallisée dans le mot d'ordre "`ilâj wa lâ izâla ${ }^{\circ}$ " (réhabilitation, non destruction), mettait fin à l'incertitude provoquée par le "provisoire qui dure ${ }^{\circ}$ ». En bute à la vindicte officielle qui les appréhendait systématiquement au seul prisme de leurs pathologies, certains de ces espaces ${ }^{13}$ furent en effet maintenus dans la précarité pendant plus de 20 ans, et exclus des - maigres dividendes étatiques.

La dynamique d'inclusion élargie de ces espaces dans le champ de l'État débute par deux décisions : leur légalisation, d'abord, et leur planification subséquente. Les initiatives civiques, toujours un peu rebelles ou soupçonnées telles, gravitant jusque-là hors du champ d'attraction de l'État, opérant souvent dans l'illégalité ou dans un état de reconnaissance tacite et donc précaire, basculent dans les réseaux du pouvoir, une fois régentées par les comités populaires (CP). Substitut fonctionnel des unités de base de l'Union socialiste et échelon terminal de l'appareil politique du régime islamiste, les CP administrent la distribution des services publics, l'érection des écoles et des hôpitaux, et servent de courroie de transmission entre les collectivités et les organisations internationales. Au total, ils prennent en charge l'intégralité de l'organisation locale dans un contexte d'État minimum (Le., État ayant déserté la fonction du service public). Ce, notamment, par la réduction drastique de la palette des produits subventionnés, le dernier étant actuellement le sucre. De plus, «'officials of the council publicly confess that the wage levels they recommanded to the state are cut by public and private employers by $25-30 \%$, whereas the recommended minimum wage constitute no 
more than $40 \%$ of the employee's subsistence requirements ${ }^{\circ}$;rade-unionist put the rate at $10 \%$ " (Awad, non publié) ${ }^{14}$. Par ailleurs, $94^{\circ} \%$ des employés de la fonction publique sont audessous du seuil de pauvreté, $61^{\circ} \%$ ont un autre métier (Sahl, 1996).

$C^{\prime}$ est afin de justifier ce paradoxal repositionnement " ${ }^{\circ}$ néo-libéral ${ }^{\circ}$ » du régime islamiste que la doctrine de l'autosuffisance (al-i'timâd 'ala-l-dhâf) a vu le jour. L'idée se résume dans cette formule lapidaire ${ }^{\circ}:$ « le local à ses citoyens, le national à l'État ${ }^{\circ}$ » (almahal li-l-muwâtinîn, al-watan li-l-dawla). La construction de mosquées, d'hôpitaux, de canalisations et de chaussées est rétrocédée, en fait comme en droit, aux juhûd dhâtiyya, au philanthropisme des marchands et aux associations locales, mais sous étroit contrôle des $\mathrm{CP}^{15}$. Ce regain de bienfaisance s'avère ainsi éminemment politique ${ }^{\circ}$ : " ${ }^{\circ}$ tout système politique, notait fort à propos Paul Veyne, tend à se transformer en gouvernement de notables et toute communauté gouvernée par des notables demandera à l'évergétisme les ressources qui lui sont nécessaires, sauf si une tradition de l'impôt existait déjà ${ }^{\star}$ (Veyne, 1976, p. $\left.{ }^{\circ} 228\right)$. Or cette tradition existe, mais faible ${ }^{16}$ et surtout massivement tendue vers l'effort de guerre ${ }^{17}$, elle laisse largement la société civile s'administrer elle-même. C'est dans ce contexte qu'il faut comprendre la projection dans le discours officiel de la notion de dhât, apanage jusqu'alors du petit peuple ou de ses édiles, et dans lequel ces derniers puisent la fierté de leur indépendance vis-à-vis de l'État. Cette projection n'est autre chose qu'un discret appel du pied à la participation informelle des citoyens à la chose publique parce que l'État, boycotté et affaibli par les ponctions militaires sur le budget, n'a pas les moyens de financer son développement. Par un étrange retournement, l'effort de guerre et l'exclusion de la scène internationale se transforment ainsi en catalyseurs du rapprochement de la société civile et de l'État.

En d'autres termes, après la parenthèse socialiste et suite à l'hémorragie budgétaire entraînée par la guerre, l'officialisation de la notion de dhât vient entériner l'échec de la politique du " $^{\circ}$ tout à $l^{\prime}$ État $^{\circ}$ » entamée par Djafar al-Nimeiry. On en revient à un contrat social plus intermédiaire où le civisme citoyen est à nouveau reconnu comme dimension constitutive de la production de l'espace public. Ce dernier redevient dès lors une instance hybride, résultat d'arrangements informels et définis au coup par coup entre l'État, les notables et les leaders associatifs locaux. Pour exemple, sur les 28 écoles de Hajj Yûsuf, le ratio dhât/État se chiffre à un tiers/deux tiers ${ }^{\circ}$ : un tiers de la facture a été payé (sans médiation par le centre) par la collectivité locale et les khayriyyîn, ces notables, tantôt anciens opposants en mal de cooptation, tantôt alliés du régime ou encore par des hommes d'affaires du Golfe. Les deux tiers restants seront payés par le ministère des Affaires d'ingénierie et par celui des Finances. L'État peut parfois financer un projet local dans son intégralité (ainsi de l'allocation - toujours à Hajj Yûsuf - de réservoirs d'eau potable), tout comme il peut se procurer les ressources nécessaires à sa propre édification, par le truchement des tributs et sans recours au circuit de l'impôt. À en croire son président, 'Abd al-Rahîm al-Muntalab, les dépenses occasionnées par la construction de la municipalité de Hajj Yûsuf (120 millions £s) ont été couvertes " ${ }^{\circ}$ sans une seule livre du gouvernement ${ }^{\circ}$ ", nous verrons sous peu de quelle manière. Le nouveau poste de police a été, lui aussi, intégralement financé «`bil'awn al-dhâtî̀, comme le proclamait avec emphase le personnage précité. Last but not least, les 88 millions Es récoltés à titre de tribut pour l'effort de guerre, viennent couronner cette relation intime, quelque peu forcée parfois, entre les ressources et les besoins de l'État et ceux des collectivités locales. 
On le voit, l'appel à l'auto-dépendance (al-i'timâd 'ala-l-dhât) renvoie moins à un désengagement de l'État vis-à-vis de ses périphéries qu'il ne motive le basculement, dans l'informel, de la dynamique d'échange et d'annexion mutuelle entre la société civile (les traditions civiques) et la société politique (les circuits de prélèvement et de redistribution du régime). Basculement qui débouche sur des pratiques redistributives populaires originales qui, bien qu'informelles, ne sont pas forcément inefficaces.

\section{Le paradoxe du sucre, ou comment la redistribution se retourne en ruse de la prévarication}

Comment financer le développement de quartiers démunis dans un contexte où l'allocation étatique de biens et services se limite, à peu de choses près, à la diffusion de sucre subventionné ${ }^{\circ}$ En retirant le sucre des mains des particuliers et en le revendant au prix du marchéo: avec les bénéfices de cette réallocation collective des subsides, on a ainsi pu construire des écoles et des mosquées. L'autogestion (i'timâd 'ala-l-dhâf) apparaît maintenant sous un jour nouveau: il ne s'agit pas d'autonomie des collectivités locales, mais d'un acte de transfert des ressources du Welfare State au profit d'investissements officieux quoique non déconnectés de l'État. En effet, les artisans de cette double opération de cession ne sont autres que les comités populaires. Comment la construction de l'école secondaire supérieure de Hajj Yûsuf a-t-elle été financée ${ }^{\circ}$ ? Une consultation de la population sous forme de conférence est organisée par la municipalité et, avec l'accord de celle-ci ${ }^{18}$, il est décidé à cette occasion de retirer le sucre subventionné à la population locale pour financer l'établissement par les bénéfices des ventes. Des experts du ministère de l'Ingénierie évaluent les frais à 220 millions £s, dont la moitié est payée par le ministère. Quant aux 110 millions restants, ils sont prélevés sur les ressources monétaires de la population locale, ressources d'ailleurs réduites aux seuls dividendes susceptibles d'être retirés de la mise en vente du sucre subventionné sur le marché libre. C'est donc le sucre qui amortit intégralement l'essentiel de la part populaire du financement. Neuf semaines de privation - de sucre - sont nécessaires pour récolter le montant requis. Ainsi, pas à pas, projet après projet ${ }^{19}$, les juhûd sha'biyya, dans la production de l'espace public local, vont venir compléter, sans se substituer à lui, l'évergétisme des notables ${ }^{20}$, inapte à lui seul à suppléer aux carences étatiques en matière d'allocation de services. De cette manière, la production de l'espace public bascule définitivement dans le champ de l'État, car si ce dernier n'en finance qu'une partie, ses agents locaux la régentent toutefois entièrement. Les comités populaires vont servir d'agent de liaison dans cette nouvelle triangulation entre les œuvres, les initiatives populaires et l'intervention étatique, dans une annexion mutuelle plus que jamais intégrée. La décision d'installer des conduites d'eau potable dans le quartier de Takâmul en est un exemple. Une conférence populaire réclamant ce projet en 1995 en est la première étape. Le $\mathrm{CP} d u$ quartier sert ensuite de relais ${ }^{\circ}$ : il transmet la $^{\circ}$ volonté populaire ${ }^{\circ}$ » au Conseil de la municipalité. La demande acceptée, le CP adresse ensuite une lettre à l'Administration des eaux qui envoie ses experts pour une estimation des coûts, établis à 170 millions Łs. Le rapport est ensuite transmis au ministère de l'Habitat qui étudie le cas et décide des modalités de contribution de l'État ${ }^{\circ}$ : il finance le projet à raison de $50^{\circ} \%$ et envoie ses experts en superviser la bonne marche. Le CP prélève le reste par la revente du sucre subventionné sur le marché en recrutant la main-d'œuvre au sein de la jeunesse locale, pour creuser le lit nécessaire à l'emplacement des conduites. En moins d'un an, un tiers 
de cet espace urbain en devenir, et peuplé de $18^{\circ} 000$ habitants, se branche sur les réseaux d'eau courante de l'État qui jusqu'alors s'arrêtaient aux abords du quartier. Un autre exemple de cette logique d'annexion mutuelle des initiatives locales est l'édification de la mairie de Hajj Yûsuf. Mais dans le duo juhûd dhâtiyya-intervention étatique, deux nouveaux éléments viennent s'ajouter ${ }^{\circ}$ les tributs imposés au peuple et l'évergétisme des dignitaires du régime. Après un meeting de la conférence réunie à l'échelon de la municipalité, un appel de dons (tabarru') est lancé et qui n'est autre chose en réalité qu'un tribut de $20^{\circ} \mathrm{Es}$, obligatoire pour toute personne inscrite sur les cartes de subventionnement. Parallèlement, les appels de dons auprès des personnalités du pouvoir (on se souvient des 5 millions offerts à titre personnel par le ministre des Finances) permettent d'accélérer la collecte de fonds ${ }^{\circ}$ : en quelques mois, les 118 millions des coûts estimés sont récoltés et six mois plus tard, en février 1996, le bâtiment officiel est sur pied, équipé en outre de 8 voitures de service.

Ces deux exemples, peut-être anecdotiques, éclairent pourtant la diversité des arrangements possibles entre les trois instances en charge de l'espace public ${ }^{\circ}$ l'État (le ministère des Travaux publics et les agents du réseau politique du régime), la contribution directe (hors les circuits de l'impôt) des citoyens ordinaires, et enfin, l'évergétisme des personnalités proches du pouvoir. Toutefois, ces exemples sont trop unilatéraux, puisqu'ils ne retiennent de cette logique intégratrice, au demeurant bien rodée, que la dimension consensuelle, laquelle s'estompe bien vite lorsqu'on regarde en détail la seconde procédure de génération de capital à partir du sucre. L'imposition de " $^{\circ}$ dons $^{\circ}$ » (tabarru'ât) à la redistribution (imposer un prélèvement comme condition d'accès au sucre subventionné) devient en effet le site d'un singulier retournement, à savoir, l'insertion de prélèvements contraints au sein des espaces du Welfare State dont il a été question jusqu'ici.

21 Les tabarru'ât, littéralement " $^{\circ}$ dons ${ }^{\circ} »$, en réalité des tributs forcés, sont au coeur de ce retournement. Cette habitante de Khartoum 3, par exemple, compte 7 personnes inscrites sur la carte de subventionnement de son foyer. Chaque semaine, en obtenant pour chaque personne son quota de sucre subventionné (450 grammes), elle économise $3^{\circ} 200^{\circ} £$ s par rapport aux frais qu'elle aurait déboursés si elle s'était approvisionnée sur le marché. Dans le même temps, ce mécanisme de Welfare State impose ses conditions ${ }^{\circ}$ : les tributs pour la collectivité ou l'État se montent par quota à... $1^{\circ} 500^{\circ} \mathrm{Es} \mathrm{s}^{21}$. Le Welfare State, dont les prélèvements informels sont ainsi compensés, devient, en définitive, un fardeau hebdomadaire d'environ $7^{\circ} 300^{\circ} \mathrm{Es}$ pour ce foyer. Quelles en sont les procédures $^{\circ}$ ? Là encore, un fonctionnement serré en réseaux permet d'assurer un prélèvement avec une évasion fiscale relativement limitée. Le sucre va en effet venir étayer une gigantesque opération de recensement de la population, mécanisme essentiel lorsqu'on se souvient avec Bayait que ${ }^{\circ}$ si la cristallisation d'un espace de domination trouve aisément ses dominants, l'enjeu est tout autant à trouver des dominés à consigner dans l'espace de domination ainsi conquis ${ }^{\circ}$ ». Le sucre en sera l'instrument': consignées dans les bureaux de l'appareil politique, les cartes de rationnement sont devenues l'oeil du pouvoir, en venant notamment informer les opérations de recrutement des conscrits potentiels et la situation financière des foyers ${ }^{22}$. Par le jeu de la carte de rationnement, et surtout par la supervision étatique de la redistribution des quotas, le sucre participe à cette opération de consignement des dominés qui procède de la cristallisation du champ étatique, au demeurant souvent léonine. Il est remarquable qu'en moins de dix ans, une mémoire de la prédation fiscale 


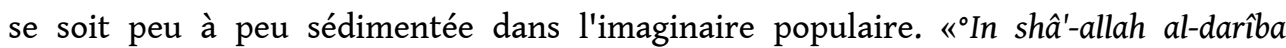
tidriba $^{\circ}$ » (si Dieu le veut, les impôts vont $\mathrm{f}$ abattre) entend-t-on parfois. Cette invective témoigne de l'appropriation, par les répertoires de l'injure, des pratiques du pouvoir accumulateur, ces pratiques qui autorisent Hamîd - poète et écrivain maintenant en exil à Doha -à qualifier le gouvernement, toujours en référence aux excès fiscaux de ce dernier, de «"Hukûmat al-faqr al-dammâr» (gouvernement de la pauvreté destructrice).

Si le sucre a servi l'enrichissement de l'État ${ }^{23}$, ses agents ne sont pas demeurés en reste, se sucrant parfois, et sans jeu de mots, allègrement au passage. Au nom du jihad, le personnel politique s'adonne en effet à la confiscation des quotas de sucre, au favoritisme dans l'allocation des revenus de la zakât, à la surestimation de l'imposition municipale sur les loyers du parc immobilier de l'État, au détournement de fonds étatiques ou au prélèvement des ressources de la population. C'est aussi à la concussion que le processus d'échanges entre sociétés civile et politique doit sa singulière vitalité. En faisant glisser les relations d'échange dans l'univers des réseaux de relations personnelles, l'expérience concrète du itimâd 'ala-l-dhât en est venue à signifier un paradoxal accroissement de la dépendance des acteurs subordonnés vis-à-vis de l'État. $\mathrm{Ce}$, par le biais des opérations d'enregistrement et des pratiques de prévarication, moralement répréhensibles, mais au rôle intégrateur certain. Par leur déploiement au sein des solidarités familiales, ethniques ou d'amitié, et via l'extension des cliques et clientèles autour des agents du pouvoir, ces opérations ont étendu l'ancrage social du régime, tant au sein de l'État que de la société dite " $^{\circ}$ civile $^{\circ} »$ - terme dont la cohérence s'est vu faiblir au fil de ces pages.

23 Le retournement des opérations de la redistribution en modalités de. prélèvement ne s'arrête pas encore lào: il aboutit, dans sa forme parachevée, à l'extorsion informelle de fonds par l'État.

\section{La part du jihad. Tributs prétoriens et rituels d'extorsion}

24 En ce matin de printemps de l'année 1997, le marché de Sajjâna est en proie à une agitation inhabituelle ${ }^{\circ}$ : en face du comité responsable de l'administration du marché, section indépendante de la municipalité de Khartoum-centre, une tente est dressée. Pas âme qui vive aux alentours ${ }^{\circ}:$ " ${ }^{\circ}$ toutes les échoppes ont été contraintes à fermer ${ }^{\circ}$ » par les Forces de défense populaire. Motif ${ }^{\circ}$ la visite du gouverneur de l'État de Khartoum, venu parler aux commerçants de jihad et de gros sous. Sous la tente, les quatre grands marchands de Sajjâna patronnent la rencontre entre les tenanciers d'échoppes et les officiels. En plus du gouverneur, le délégué du président du Conseil municipal et l'intégralité des 15 membres du comité du marché sont présents sur l'estrade. Deux d'entre eux appartiennent de surcroît au Conseil municipal. L'enjeu de la rencontre peut se lire dans l'intérêt accru du régime pour les marchés comme source majeure de revenus pour l'État. Au diapason de cet intérêt, le gouverneur se livre depuis quelques temps à des tournées de prélèvement dans les trois marchés que compte Khartoum $3^{\circ}$ : le souk populaire, le souk 'Abd al-Mun'im, le souk al-Sajjâna. Aujourd'hui, il se trouve dans le dernier de ces souks, escomptant réunir 250 millions £s, et obtenir le soutien des quatre grands commerçants évoqués. Ces derniers sont fidèles aux injonctions du régime car il en va de leur intérêt ${ }^{\circ}$ : ils lui sont redevables d'une part notable de leur richesse et savent que leurs recettes futures dépendent étroitement de leur bonne entente avec les agents du pouvoir. Il leur faut en particulier l'appui de ceux qui ont des connexions avec la branche de la Chambre des impôts (Diwân al-Darâ'ib) dans le marché, 
appui essentiel pour l'accès aux licences de commerce. Il leur faut également ménager le personnel des huit banques islamiques du souk de Sajjâna (qui compte onze établissements financiers au total), banques dont le personnel dirigeant est constitué de personnalités proches du FNI, site vital cette fois pour l'accès au crédit ${ }^{24}$. Cette double dépendance par rapport aux licences et aux crédits, dont la distribution est maintenant sous contrôle étroit du régime (via ses agents au sein des municipalités et des (P), y est sans doute pour quelque chose dans l'enthousiasme que les grands commerçants témoignent à l'égard du jihad. Sur la table du gouverneur, chacun d'eux versera 4 millions Es en espèces, lesquels seront rapidement scellés dans des sacs prévus à cet effet et remis aux soldats accompagnant l'officiel.

La visite du gouverneur représentant d'abord une menace financière (il reste encore 234 millions de Es à renflouer), la plupart des commerçants présents dans l'assemblée adoptent un profil bas ${ }^{\circ}$ : tous inscrits au comité du marché, leurs noms figurent sur des listes détenues par les membres de la municipalité et des comités populaires. Compte tenu de leur tiédeur à l'égard du jihad et de son financement, il est donc décidé, à la place d'une collecte collective, de procéder sur une base individuelle en s'appuyant sur les informations personnelles contenues dans le fichier du comité du marché.

Le quatuor évoqué mène la "'délégation ${ }^{\circ}$ ", encadré de soldats, de l'officier administratif de la municipalité (agent de liaison entre le comité du marché et la municipalité) ainsi que de son directeur exécutif et de deux membres de la municipalité. CM. se souvient encore de leur arrivée dans son échoppe, et de la façon dont, à grands renforts de devises religieuses, ils lui ont demandé quelle part il entendait verser au jihad. Il n'a pas répondu tout de suite et cette hésitation lui a été fatale ${ }^{\circ}$ : sa dîme a été fixée de force par un des membres de la municipalité et d'emblée enregistrée par le secrétaire du comité. Ainsi ce jour-là, de proche en proche, 200 millions $£ s\left(130^{\circ} 000^{\circ} \$\right)$ ont été captés par l'effort de guerre et l'État à Sajjâna.

Loin de constituer une simple anecdote, cet événement est désormais le pain mensuel des marchands, boutiquiers et petits entrepreneurs du marché. Non content de sa récolte, le gouverneur revient peu après réclamer une somme équivalente, essuyant cette fois-ci un refus net des autorités locales, avec à leur tête, le directeur exécutif de la municipalité, ancien associé de prélèvement du gouverneur. Mais cette rebuffade constitue l'exception et non la règle ${ }^{\circ}$ : dans l'ensemble, les souks obtempèrent sans trop de heurts à l'appel - de fonds - du jihad. Durant le même mois, toujours à Khartoum, 3,5 milliards £s, soit 2,3 millions \$, sont récoltés au marché populaire, le centre commercial du quartier. Ces collectes sont vertigineuses dans les marchés majeurs de la capitale, notamment au marché libyen, où ce ne sont pas moins de 6 milliards £s, soit 4 millions \$, qui sont récoltés ce jour de juillet 1997, pour être convertis en fusils.

$\mathrm{Au}$ marché 'Abd al-Mun'im, les rituels d'extraction au profit du jihad corroborent les procédures observées à Sajjâna. Là aussi, tout se déroule sous le suwân, la tente traditionnelle des cérémonies, dressée après que des employés affectés à la municipalité eurent déblayé le souk. La raison': «`al-Wâlî jây», le gouverneur arrive, appuyé, comme de coutume, par le bureau exécutif de la municipalité et entouré, par ailleurs, des commerçants du marché liés au régime ainsi que du comité du marché. Toutes les échoppes sont fermées durant la cérémonie d'évulsion monétaire. Les cent commerçants du marché sont là à écouter en silence le premier discours tenu par le président de la municipalité prêchant pour le jihad. Le responsable de la division " ${ }^{\circ}$ mobilisation politique et propagande ${ }^{\circ}$ " (ta'bi'a siyâsiyya wa da'wa) du comité du 
marché lui succède à la tribune, appelant encore une fois les commerçants à contribuer à l'effort de guerre. En clôture, reconnaissant devant la générosité (à venir) des échoppiers du marché, le gouverneur vante la loyauté et la solidarité des marchands. Les sacs préparés par la municipalité n'attendent plus que les versements des premiers donateurs. Le jour suivant, la même délégation va rendre visite aux marchands timorés, comme elle a désormais l'habitude de le faire tous les deux ou trois mois, requérant des sommes dont le montant varie au gré de ses passages et oscillant entre 75 et 150 millions Es.

Notre intuition première se précise ${ }^{\circ}$ : les donations - ici destinées à l'effort de guerre, mais pas uniquement - ont certes pour rôle premier de renflouer les caisses de l'État parce que l'imposition fiscale ne suffit pas. Au-delà, et de manière involontaire, la mise en scène et la gestion du prélèvement informel soutiennent deux logiques d'intégration. L'une est infra-étatique et relève de la fusion organique entre l'appareil politique (les municipalités et le comité du souk qui leur est affilié) et l'administration (tantôt le bureau des impôts, tantôt le ministère des Affaires d'Ingénierie ou encore celui des Finances). L'autre concerne le resserrement des liens entre la société civile ici, le monde des marchés - et la société politique. Un resserrement qui s'exprime évidemment dans le domaine des transferts de fonds, mais également dans celui de l'information récoltée durant les cérémonies extractives où les allégeances sont matériellement cristallisées en termes de dons ${ }^{\circ}$ : les fidèles apparaissent au grand jour grâce aux sommes enregistrées dans la comptabilité du secrétaire du comité, alors qu'à la limite, les marchands trop pusillanimes s'exposent de facto aux représailles fiscales des agents du régime. Une fois de plus, les $\mathrm{CP}$ apparaissent en première ligne de la dynamique d'intégration politique et fiscale du souk, deux dimensions qui sont désormais, et plus que jamais, indissociables dans ce processus $d^{\prime}$ " ${ }^{\circ}$ annexion mutuelle ${ }^{\circ} \gg$ des sociétés civile et politique. Ils ont immanquablement des représentants dans les comités des marchés, ce qui permet de gérer les conflits au sein de la classe marchande, clairement polarisée aujourd'hui entre les supporters du FNI et les outsiders de l'ordre politique actuel, bien souvent des sympathisants des anciens partis Umma et Unioniste. M. K., du souk 'Abd al-Mun'im, un vieux fidèle du Parti unioniste a été vaincu après une longue guerre d'usure avec l'administration, lorsqu'il a reçu une facture de 18 millions £s à régler. A. T. ou encore W. T. - tous supporters du Parti unioniste ou du Parti Umma -connurent le même sort au marché al-'Arabî, expulsés de la place à coups $\mathrm{d}^{\prime}$ ' $^{\circ}$ attaques fiscales ${ }^{\circ}$ » (hujûmât darîbiyya) et $\mathrm{d}^{\prime}$ ' $^{\circ}$ obstructionnisme procédurier ${ }^{\circ} »\left(t^{\prime}\right.$ wîq $\left.i j r \hat{a}^{\prime} \hat{\imath}\right)$ selon les termes utilisés par un marchand unioniste, comme eux " $^{\circ}$ vaincu à coups de taxes et de refus de licences de commerce ${ }^{\circ}$.

Sont emblématiques de cette polarisation de la classe marchande, le schisme survenu dans le marché 'Abd al-Mun'im et les modalités de sa progressive résorption. La première aile, créée par les juhûd dhâtiyya d'un groupe de commerçants, sur le terrain offert par 'Abd al-Mun'im Hassan à la collectivité locale en 1946, se politise en 1973 avec l'arrivée de marchands déplacés du centre-ville. Le responsable de ce transfert en est la présidente du Conseil municipal de l'époque, sympathisante notoire du Parti unioniste dont elle fut le premier membre féminin, ce qui explique - mais nous sommes dans l'ordre des présomptions - le fort taux d'unionistes et de mahdistes qui s'établissent alors dans le petit marché de Khartoum 3. À la même époque, une extension est créée et le souk 'Abd al-Mun'im est alors rebaptisé souk al-Inqâdh (marché du salut), et peuplé de marchands idéologiquement conformes à la nouvelle appellation du lieu. Dans la même période, plusieurs commerçants de l'ancienne aile 
sont contraints de fermer boutique. Les alliés du régime mettent la main sur le souk, évinçant la première génération de marchands qui abdiquent, l'un après l'autre, sous le coup des pressions fiscales et administratives. À l'entrée, deux commerces présentent portes closes ${ }^{\circ}$ : deux anciens, deux faillis. En vis-à-vis, un autre déplacé de la première génération a également fermé boutique, alors qu'à l'angle de la rue, la devanture flambant neuf d'un nouvel arrivant réputé proche du régime, côtoie celle, usée, d'un vieux supporter déclaré du Parti unioniste, arrivé en 1973 lors du transfert du marché du centre-ville. Ce tandem symbolique de la passation des pouvoirs entre l'ancienne classe commerçante et l'élite progouvernementale a affecté l'ensemble de la nation, et s'est traduit, en termes concrets à Sajjâna, par 12 cas de faillite ces dernières années, la plupart faisant suite à des taxation indues, des blocages administratifs répétés ou à la fermeture de l'accès au réseau bancaire.

Les rituels du tribut contraint au marché de Sajjâna et la visée hégémonique des marchands frontistes sur le marché 'Abd al-Mun'im décrite ci-dessus, permettent d'émettre quelques propositions générales sur la teneur de cette dynamique que nous avions désignée, en termes lâches, par " $^{\circ}$ annexion mutuelle ${ }^{\circ}$ » des sociétés politique et civile.

D'abord, il devient évident que celle-ci ne fait pas l'économie de la contrainte ou, à tout le moins, de la prévarication, et ceci de manière identique auprès des marchands ou des individus ordinaires. Au niveau de l'extraction de ressources monétaires, excepté le tribut librement consenti des personnalités proches du régime (sur la base de contreparties sous forme de licences, de réductions ou d'exemptions fiscales, ou d'accès au crédit bancaire), le prélèvement s'effectue sous la forme de $^{\circ}$ donations ${ }^{\circ}$ » (tabarru'ât), contraintes et organisées par les agents du système politique ${ }^{25}$ aux trois niveaux du gouvernorat, de la municipalité et du quartier. Protocolaire et ritualisée en général, cette extraction n'oblitère pas les initiatives spontanées ${ }^{\circ}$ : les marchands du marché local de Khartûm al-Jadîd n'oublieront pas de sitôt ce jour noir où le président de la municipalité pris seul le volant du pick-up officiel pour récolter sur le champ auprès des boutiquiers 30 millions $€ s$ au nom du jihad, dont 15 seulement seront rétrocédés au ministère des Finances ${ }^{26}$. La cristallisation de l'État, loin d'être ce processus lisse et téléologique de complexification des appareils politiques, voués immanquablement à plonger leurs réseaux toujours plus loin dans le tissu social, fait la part belle aux rapports de force, aux stratégies d'influence et d'exclusion. L'annexion mutuelle tient en définitive moins de la fusion harmonieuse que de l'absorption autoritaire d'une partie du champ social (i.e., d'un prélèvement sans consensus minimum) par l'exploitation systématique des outsiders des réseaux clientélistes du pouvoir.

33 En second lieu, il convient $\mathrm{d}^{\prime}$ insister sur l'ubiquité du système politique ${ }^{\circ}$ : les membres du FNI opèrent au sein d'un réseau infra-étatique ramifié mais non hégémonique (tout le personnel du système politique ne leur est pas acquis, nous y reviendrons), étayé sur un dense lacis de relations personnelles et capable de transcender les strictes barrières de l'appartenance à l'État. Cet État, que le FNI déborde sans contenir, tire sa force de son informalité, de l'étendue et du caractère ramifié des liens personnels qui compensent partiellement l'inachèvement du champ institutionnel. À Sajjâna, le régime trouve en dehors des membres de la municipalité et des $\mathrm{CP}$, l'appui du quatuor évoqué dont deux membres seulement ont une appartenance politique déclarée. Par la présence de deux membres du CP et celle de l'officier administratif enfin, le pouvoir 
peut compter au sein du comité du marché, formellement indépendant du système politique, des individus lui étant acquis ${ }^{27}$. Un comité qui constitue, au demeurant, un excellent exemple des logiques d'absorption de la société civile par le régime ${ }^{\circ}$ : ${ }^{\circ}$ au début, le comité avait été fondé pour servir les marchands, maintenant c'est devenu un simple moyen de contrôle pour le gouvernement ${ }^{\circ} »$, déclarait un de ses membres, reprenant quasi à l'identique, des propos maintes fois entendus au sujet des $\mathrm{CP}^{\circ} \mathrm{Au}$ début en effet, le comité s'est constitué sans aucune supervision de l'État. Après la Révolution du Salut, il est formellement enregistré à la municipalité. D'emblée, deux divisions sont ajoutées ${ }^{\circ}$ : ${ }^{\circ}$ mobilisation politique et propagande ${ }^{\circ}$ » (ta'bi'a siyâsiyya wa $\left.d a^{\prime} w a\right)^{28}$, et " $^{\circ}$ sécurité et ordre public ${ }^{\circ}$ ( al-amn wa-l-nidhâm al-'âm) - divisions que l'on retrouve par ailleurs à tous les échelons de l'appareil politique, y compris dans les $\mathrm{CP}$ qui lui sont organiquement liés.

Nous étions partis de la charité et de la bienfaisance civile et nous voici parvenus au cœur de leur antithèse ${ }^{\circ}: l^{\prime}$ extorsion étatique de fonds. Un renversement qui trouve ses conditions de possibilité dans l'ambivalence même des répertoires de l'islam où les espaces de la redistribution et de la solidarité ont servi à la mise en place d'un système de domination fondé sur un programme ultra-libéral qui n'a rien à envier aux recettes des " $^{\circ}$ reaganomics ${ }^{\circ}$ - idéologiquement pourtant aux antipodes du régime actuel.

\section{Chimères de l'utopie révolutionnaire ${ }^{\circ}$; de quelques syncrétismes islamistes du politique}

Dans le contexte de démonétisation actuel du régime soudanais, les formules rapides du type " $^{\circ}$ arabic islamic theocracy» (Frazer, 1996, p. $\left.{ }^{\circ} 10\right)$, concomitantes de sa mise à l'index dans le nouvel ordre mondial, masquent une formule de gouvernement autrement plus complexe qui n'échappe pourtant pas - à l'image des autres gouvernements à vocation islamiste (Bayait, Roy et Adelkhah, 1993) -aux déterminations de son histoire politique et de son environnement international.

De fait, dans son expérience immédiate, et malgré sa mobilisation des répertoires islamistes, le régime soudanais n'est pas complètement hermétique aux problématiques politiques du nouvel ordre mondial ${ }^{29}$. De même, ne répugne-t-il pas à recourir aux méthodes de gouvernement éprouvées par ses prédécesseurs, lorsqu'au diapason continental, l'hymne au " ${ }^{\circ}$ peuple ${ }^{\circ}$ » supplantait encore les références au Coran dans les discours officiels. Ce double code idéologique n'a de cesse d'interférer sur la pratique actuelle du pouvoir et aura raison du dessein révolutionnaire professé par les têtes pensantes du régime actuel. L'exceptionnalité de l'expérience islamiste du pouvoir s'est dissoute dans l'ordinaire de la globalisation et de son histoire, une fois que ses catégories, placées " $^{\circ}$ en relation ostensible avec le monde ${ }^{\circ}$ » (M. Augé), se sont gorgées de la vulgate néo-libérale de son environnement international, avant de s'imprégner de plus d'un scénario nimeiriste du politique.

Toutefois, loin de constituer l'ultime révélateur de "l'échec de l'islam politique ${ }^{\circ}$ » (Roy, 1992), cette dépendance du système politique islamiste, aussi bien à l'égard du «'temps du monde ${ }^{\circ}$ » que des " $^{\circ}$ nappes d'histoire lente ${ }^{\circ}$ " (Braudel, 1935, $\mathrm{P}^{\circ}$ 83) de la trajectoire soudanaise du politique, sous-tend davantage des dynamiques d'innovation qu'elle n'oppose de résistance à son historicité. Tout au long de cet article, nous avons vu les lignes de continuité et les facteurs exogènes interférer en permanence dans la 
cristallisation de l'État islamiste. L'appellation d'origine " ${ }^{\circ}$ Révolution du Salut ${ }^{\circ}$ » (thawrat al-inqâdh) ne saurait faire illusion sur ce qui se trame réellement dans l'expérience islamiste du pouvoir ${ }^{\circ}$ : le régime actuel s'est construit aux antipodes d'une rupture brutale avec les formules politiques du monde extérieur (africain ou international) ou de son passé immédiat (post-colonial), rupture qu'il appelait pourtant de ses vœux. En lieu et place du " ${ }^{\circ}$ tout à l'islam ${ }^{\circ}$ » c'est plutôt un scénario syncrétique qui s'est dévoilé au long de ces quelques pages où s'entrelacent trois thématiques du politique $^{\circ}$ : islamiste, populiste et libérale.

Premier référentiel mobilisé par le régime, l'islam, s'il est le plus visible, n'est pas forcément le plus structurant. Dans une certaine mesure, il a servi des logiques distributives, notamment par le biais de la zakât, de la caisse de solidarité sociale, des multiples organisations de soutien (aux martyrs, aux familles, aux étudiants, aux familles des victimes de guerre) tout comme il a servi de motif aux multiples œuvres pieuses qui, aux marges de l'État, se sont historiquement et intimement liées à son développement en suppléant peu ou prou aux carences de ce que les experts du FMI appellent aujourd'hui le ${ }^{\circ}$ safety net ${ }^{\circ} \|^{30}$. En ce sens, l'innovation religieuse fut bien une des matrices de la modernité étatique.

De manière autrement plus magistrale en revanche, la référence à l'islam est venue renforcer les logiques de concentration et d'accumulation du capital entamées dès l'indépendance, en servant de registre unificateur à la classe dominante en formation ${ }^{310}$ : cette dernière trouve dans les recettes néo-libérales un moyen remarquable d'accumulation de ressources économiques et politiques. Sous la pression du nouveau « ${ }^{\circ}$ lobby $\mathrm{FMI}^{\circ}$ » (Awad) solidement ancré en son sein, le régime n'hésite pas à faire diminuer l'impôt sur les hauts revenus de $60^{\circ} \%$ à $30^{\circ} \%$. Cette volonté hégémonique néo-libérale des dirigeants se voit confirmée par un processus de privatisations entamé de manière singulièrement rapide: 60 entreprises ont été privatisées (entièrement ou en partie) en l'espace de deux ans, la plupart des cessions ayant été vendues - souvent aux dirigeants du $\mathrm{FNI}^{32}$ - à des seuils bien en deçà des recommandations des experts. Les profits immédiatement dégagés le sont par simple hausse des prix sur des biens jusqu'alors publics. Les opérateurs économiques du régime se sont ensuite transformés en maîtres $d u$ ${ }^{\circ}$ chevauchement ${ }^{\circ} »$, usant de leur position dans l'appareil d'État pour s'accaparer graduellement des secteurs entiers de l'économie ${ }^{\circ}$ : mainmise sur l'allocation des licences à l'exportation (qui leur permet de réduire drastiquement les opérations de leurs concurrents), contrôle complet sur le secteur bancaire, notamment les banques islamiques étrangères gérées en partenariat avec des agents du pouvoir local. Pour clore, on ajoutera que cette hégémonie fut encore renforcée par la possibilité de contourner l'imposition fiscale en recourant aux institutions de charité exemptées de taxes. Sous couvert de sadâqa et de takâful, ces dernières permettent de banales opérations marchandes sans rapport aucun avec un quelconque objectif caritatif. Les institutions homologuées par les injonctions islamistes de solidarité sociale se transforment de la sorte en l'un des plus performants leviers de la concentration du capital aux mains de l'establishment islamiste ${ }^{33}$.

En second lieu, les références au jihad ou à la zakât ont facilité - ou couvert -la mise en valeur fiscale de la société par des prélèvements massifs en faveur de l'effort de guerre $^{34}$. Grâce à ce dernier, et selon les bonnes règles de la " ${ }^{\circ}$ politique du ventre ${ }^{\circ}$ ", plus d'une personnalité officielle a trouvé de quoi se " $^{\circ}$ nourrir ${ }^{\circ} »^{35}$ au passage, comme le rappellent les « ${ }^{\circ}$ virées extractives ${ }^{\circ}$ » du président de la municipalité de Khartoum sud. 
La réforme de l'impôt a ensuite permis une imposition élargie de la société en étendant la base de la taxation directe jusqu'à atteindre le secteur informel du petit artisanat et des productions domestiques. Aujourd'hui, même les vendeuses de thé dans les rues n'échappent pas au champ fiscal d'un État, décidément plus libéral à l'égard des hauts revenus que des petits.

Quant à la référence au dhât, ce label d'extranéité de la société par rapport à l'État, elle n'est guère qu'une rhétorique au service d'un projet néo-libéral renvoyant la société civile à ses propres moyens (Abdou Maliqalim, 1994). Pour le reste, la tentation

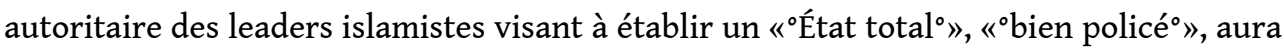
bientôt raison de cette inclination libérale du régime ${ }^{\circ}$ : les espaces d'extranéité par rapport au champ du pouvoir au sein de la société civile ont été confinés par le gouvernement à la sphère du privé ${ }^{36}$, alors que les entreprises civiques, qui ont traditionnellement contribué à la production de l'espace public, ont été absorbées par les réseaux du pouvoir et souvent mises au profit de l'accumulation personnelle de ses membres, perdant dans la foulée leur vertu intégratrice. Les $\mathrm{CP}$, comme les unités de base de l'Union socialiste, présentent, quant à eux, un bilan intégrateur bien mince ${ }^{\text {: }}$ " ${ }^{\circ}$ They played only a marginal role in conveying popular concerns in the national leadership or in politicising the population in the manner intended. ${ }^{\circ}$ (Niblock, 1987, p. ${ }^{\circ} 270$ )

Enfin, et l'essentiel est sans doute là, au-delà de la clarté des références idéologiques, l'État islamique doit aussi sa cristallisation à des procédures silencieuses, sans références discursives, au sein desquelles on trouve au premier chef l'innommé de la coercition et de la prévarication, dont les islamistes ne semblent guère plus avares que leurs prédécesseurs. La domination continue à s'exercer sans ambages, à peine atténuée par la relative intimité qui unit les membres du champ politique à leur environnement social et notamment aux membres de leur opposition traditionnelle ${ }^{37}$, et certainement élargie par les nouvelles technologies de contrôle. Nous avons évoqué le travail infligé aux jeunes des quartiers populaires au nom de l'effort civique, l'évacuation manu militari de quartiers informels ${ }^{38}$. On y ajoutera, monnaie courante désormais dans les marchés, les rafles du percepteur, du président du Conseil municipal et autres $^{\circ}$ attaques fiscales ${ }^{\circ}$ » et " $^{\circ}$ obstructionnismes procéduriers ${ }^{\circ}$ », infligés à la " ${ }^{\circ}$ bourgeoisie non capturée ${ }^{\circ}$ " et exclue des groupes de commerçants cristallisés autour des leaders politiques locaux ou nationaux. Plus banal encore au registre de la coercition': l'allocation autoritaire de valeurs (la plus problématique étant celle qui concerne la pudeur) ou de codes symboliques discriminants (en particulier vestimentaires, comme l'obligation du port du foulard qui le dispute aux uniformes scolaires modelés sur l'habit militaire) ou encore la conscription contrainte. Cette enfilade à peine polémique de constats entrevus au long de ces quelques pages nous renvoie à notre point de départ ${ }^{\circ}$ : l'État est imposé, y compris au sens le plus littéral du terme, à une population qui se dit elle-même ta'bâna (fatiguée), et mukta'iba (lassée, déprimée) de son rapport à ce dernier.

43 Ces constats permettent ensuite de resituer l'expérience de l'État islamique dans sa banalité dès lors qu'il faut bien admettre avec Jean-François Bayart (1987, p. $\left.{ }^{\circ} 300\right)$ qu'ils valent pour l'ensemble du continent africain et au-delà. Reprenant sous une forme plus autoritaire les formules politiques antérieures, les dirigeants islamistes ne sont parvenus à dépasser ni les déterminations liées à l'insertion de leur État dans le champ international, ni celles provenant de leur historicité propre, populiste et prétorienne. En dépit du tandhîm shumûlî, le «'système intégral ${ }^{\circ}$ » revendiqué par les idéologues du 
régime actuel, l'État islamiste reste un éternel produit de synthèse, obéissant, et fidèle en cela à ses prédécesseurs, à une règle de l'inachèvement et de l'indétermination. Comme le fleuve d'Ovide - et peut-être trouve-t-il là le secret de sa longévité - il continue de puiser à la source de référentiels multiples, une diversité nullement tarie par les lancinants rappels au Coran et à la sharia lancés par le nouveau régime. Au total, la formule islamiste du politique, de saga révolutionnaire qu'elle a pu être à ses débuts, a bien vite " "réinventé sa banalité"», promptement retournée en vecteur, à peine drapé des références à la shûra et à la sharia, des permanences de la construction métisse de l'État postcolonial sub-saharien, et massivement irriguée désormais, comme le reste du continent, par des thématiques néo-libérales sur le pouvoir et l'État.

\section{BIBLIOGRAPHIE}

ABDELAZIZ Azza Ahmed, 1997, «"L'impôt religieux au Soudan: la zakât ${ }^{\circ} »$, Les Notes du Poste d'expansion économique et commerciale de Khartoum, Khartoum, Poste d'expansion économique et commerciale.

ABDOU MALIQALIM Simone, 1994, In whose image? Political Islam and Urban Practices in Sudan, Chicago, University of Chicago Press.

AWAD Mohamed Hachim, Poverty in Soudan: Anatomy and Prognosis, non-publié.

BAKHEETAdil Hassan, $\ll^{\circ}$ The Role of Sudanese Social Funds in Poverty Alleviation. An Evaluation of Zakât Fund Performance ${ }^{\circ}$ », UNDP-Friedriech Ebert Stiftung Seminar, «'Poverty in Soudan, Towards an agenda for research ${ }^{\circ}$, Khartoum University, 23-24 ${ }^{\circ}$ août 1997, communication non publiée.

BAYART J.-F, ROYO., ADELKHAH F., 1993, Thermidor en Iran, Bruxelles, Complexe.

BAYART Jean-Francois, 1987, L'État en Afrique. La politique du ventre, Paris, Fayard.

BRAUDEL Fernand, 1985, Écrits sur l'histoire, Paris, Flammarion.

FRAZER Lawrence ${ }^{\circ}$

- 1995, «"The Orwellian Sate of Soudan», The Economist, 24 juin.

- 1996, «Fragmentation of the State ${ }^{\circ} »$, Civil Society, mai.

HAENNI Patrick ${ }^{\circ}$ :

- 1996, « ${ }^{\circ} \mathrm{Charité} \mathrm{et} \mathrm{politique,} \mathrm{le} \mathrm{don} \mathrm{dans} \mathrm{la} \mathrm{classe} \mathrm{d'affaire} \mathrm{cairote}{ }^{\circ} »$, Egypte-Monde arabe, vf 25.

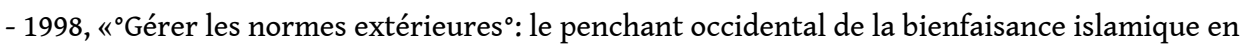
Egypte ${ }^{\circ} »$, Egypte/Monde arabe, $\mathrm{n}^{\circ} 30-31$.

HANNA Nelly, 1997, Making big money in 1600: Biography of Abou Tekkeyia Shahbandar al-Tujjar, Cairo, AUC Press.

HAYNES Douglas, 1967, « $^{\circ}$ From Tribute to Philanthropy, the Politics of Gift Giving in a Western Indian City ${ }^{\circ}$, Journal of Asian Studies, vol. ${ }^{\circ} 46, \mathrm{n}^{\circ}{ }^{\circ} 2$. 
KHOURI-DAGHER Nadia, " $^{\circ}$ The answers of civil society to a defaulting state ${ }^{\circ}$ :a case study around the food question in Egypt ${ }^{\circ} »$, Middle East Studies Association, 15-17 novembre 1987, communication non publiée.

MARCHAL Roland, 1992, « ${ }^{\circ}$ Le Soudan entre islamisme et dictature militaire ${ }^{\circ}$, Maghreb-Machreck, $\mathrm{n}^{\circ \circ} 137$.

NIBLOCK Tim, 1987, The dynamics of Sudanese politics 1898-1985, MacMillan Press.

ROY Olivier, 1992, L'Échec de l'islam politique, Paris, Seuil.

SAHL Ibrahim Mohamed Gum'a, 1996, From elitism to poverty: coping and survival strategies of the Sudanese "Middle class $^{\circ} »$ in the public sector, Thesis, University of Khartoum, Department of Economics.

TORE Nordenstam, 1968, Sudanese ethics, Uppsala, The Scandinavian Institute for African Studies.

VEYNE Paul, 1976, Le pain et le cirque. Sociologie d'un pluralisme politique, Paris, Seuil.

\section{NOTES}

1. Laissant Habermas à son Agora, nous parlerons d'espace public dans son sens le plus immédiat d'espace ouvert à tous, ou d'institutions établies pour les besoins de la population ${ }^{\circ}$ : routes, hôpitaux, lieux de culte, mais aussi canalisations d'eau potable et conduites sanitaires entrent dans cette catégorie.

2. En nous inspirant librement de Paul Veyne (1976), nous définirons l'évergétisme comme

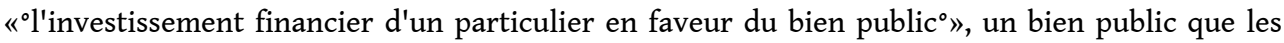
agents de l'État sont les premiers à définir en fonction de leurs stratégies de pouvoir, et parfois comme c'est le cas au Soudan - de guerre. En vertu de cette définition, l'évergétisme est susceptible de revêtir les deux formes de la philanthropie sociale ou du tribut financier en faveur des opérations civiles et militaires de l'État.

3. Du point de vue de la religion, elle repose sur trois termes, peu ou prou rattachés au devoir de solidarité et d'entraide ${ }^{\circ}$ : Sadâqa, takâfui, zakât. Pour plus de détails sur les réalisations et l'usage politique contemporain de ces représentations religieuses, voir notre article (1996). Sur l'expérience soudanaise de ces catégories ${ }^{\circ}$ : Abdelaziz (1997) ${ }^{\circ}$; Awad $^{\circ}$; Tore (1968, notamment le chapitre 7 sur l'ethos de la générosité) ${ }^{\circ}$; Bakheet.

4. Une expression qui renvoie aussi bien à ces marchands avides de licences et de réductions ou d'exemptions fiscales - négociant celles-ci à coups d'investissements philanthropiques - qu'aux comportements sécuritaires vis-à-vis de l'arbitraire fiscal -arbitraire dont on reparlera.

5. Pour quelques références comparatives, voir Hanna (1997) ${ }^{\circ}$; Haynes (1987) ${ }^{\circ}$; Haenni (1998).

6. Cette participation informelle des individus à la chose publique que désigne le terme de juhûd dhâtiyya, ou initiatives civiques, recouvre deux ordres de pratiques ${ }^{\circ}$ les œuvres philanthropiques d'abord, allocation de ressources dyadique et verticale (entre le prestataire et les bénéficiaires) d'un particulier à la collectivité locale ou nationale (et donc potentiellement de l'État) ${ }^{\circ}$; les initiatives populaires ensuite, qualifiées par différenciation de juhûd sha'biyya, menées par des dirigeants associatifs de même extraction sociale que les bénéficiaires de leurs actions.

7. Le même auteur en fut apparemment bien conscient, lorsque, réévaluant sa première analyse de l'État, il publie ${ }^{\circ}$ : «'Fragmentation of the State ${ }^{\circ} »$, Civil Society, mai ${ }^{\circ} 1996$. L'ambivalence du régime actuel qui est ainsi soulignée par Frazer dans cet article et dans l'article précité (1995) sera également notre perspective.

8. Par ce terme, nous entendons ces transferts volontaires ou contraints, mais toujours informels, des ressources (monétaires ou autres) de la société civile en faveur du pouvoir. 
9. Le coût quotidien de la guerre est actuellement estimé à 2 millions \$.

10. Selon notre définition (voir note 2), lever une armée relève bien de l'évergétisme (et par inclusion, des juhûd dhatiyya), dès lors qu'une telle conduite concorde avec l'intérêt public tel qu'il est défini par les dirigeants (la participation au jihad est un devoir de citoyen).

11. Babikeur Adam de son vrai nom, modeste marchand à ses débuts, a d'abord fait fortune dans le commerce de devises. Sans être militant du régime, il " $^{\circ}$ compense $^{\circ}$ » son non-engagement politique par une pratique constante du tribut, seul moyen véritable pour lui de se doter d'appuis dans l'administration nécessaires à la bonne marche de ses affaires.

12. $1^{\circ} 500^{\circ} \mathrm{Es}$ équivalent environ à $1^{\circ} \$$.

13. Parfois définis comme « ${ }^{\circ}$ lieux du crime ${ }^{\circ} »$ (majâl al-ijrâm), de la pauvreté, des luttes intestines et tribales, des épidémies et de l'arriération (takhalluf).

14. Le salaire minimum a été fixé en 1996 à $18^{\circ} 000^{\circ} \mathrm{Es}$ par mois, soit $11,5^{\circ} \$$.

15. Lesquels administrent jusqu'aux affaires courantes des khilwa, ces petites écoles coraniques, œuvres des notabilités locales.

16. La part des impôts (sur les bénéfices, les fortunes et les revenus) dans le budget au Soudan s'élevait à $18^{\circ} \%$ en 1987 alors que le taux moyen des pays en voie de développement s'élevait à $22,3^{\circ} \%$ des revenus de l'État pour la même année.

17. C'est une des critiques régulières adressées à la zakât qued'être trop orientée sur le jihad, au détriment de ses autres catégories de bénéficiaires.

18. La transparence de l'opération est toutefois sujette à caution. En contradiction avec le récit du président du $\mathrm{CP}$, certains démentiront qu'il y ait eu accord, parlant plutôt de «ĳmâ' sukût!», de ${ }^{\circ}{ }^{\circ}$ consensus silencieux ${ }^{\circ} »$, et affirmant que seuls les proches du régime ont été convoqués.

19. La liste des priorités a été par exemple établie de la façon suivante par le CP de Takâmul, une des sous-divisions de la municipalité de Hajj Yûsufo: dès 1989, installation des premiers centres de soin, encore embryonnaires et sans bâtiments en dur ${ }^{\circ}$; les années 1992-1993 sont consacrées à la planification urbaine et à l'allocation des parcelles aux particuliers ${ }^{\circ}$; à partir de 1994, construction d'écoles ${ }^{\circ}$; dès 1995, installation de conduites d'eau ${ }^{\circ}$; en 1996 enfin, le premier poste de police est installé, toujours aux frais de la population.

20. Nous avions évoqué, en début d'article, les œuvres édilitaires réalisées dans le quartier.

21. Cette somme comprend notamment ${ }^{\circ}$ : le traitement des ordures (500 Es/semaine), la lutte contre la malaria $\left(500^{\circ} \mathrm{Es} / \mathrm{mois}\right), \mathrm{l}^{\prime}$ aide aux Forces de la police populaire $\left(500^{\circ} \mathrm{Es} / \mathrm{mois}\right), \mathrm{l}^{\prime}$ aide à la télévision $\left(500^{\circ} \mathrm{Es} / \mathrm{mois}\right)$, le soutien à l'éducation $\left(500^{\circ} \mathrm{Es} / \mathrm{mois}\right)$. Les sommes récoltées sont versées au ministère des Finances via les unions coopératives et les CP Il est à noter que ces contributions informelles ne sont pas une alternative au prélèvement officiel (taxes annuelles et $\mathrm{za} / \mathrm{câf}$ ), lequel continue d'accroître son rayonnement (voir conclusion) grâce aux licences de commerce et de véhicules. Ce dernier se chiffre par quota $\mathrm{à}^{\circ}: 2^{\circ} 000^{\circ} \mathrm{Es}$ pour les Forces populaires de défense $\mathrm{o}^{\circ} ; 2^{\circ} 000^{\circ} £$ s pour les victimes de guerre ${ }^{\circ} ; 3^{\circ} 000^{\circ} £$ s pour l'effort de guerre et $10^{\circ} 000^{\circ} £ \mathrm{~s}$ pour la zakât. A ceci s'ajoutent enfin les impôts et la zakât sur les revenus.

22. Dans le cadre de l'allocation de la zakât, des enquêtes sont en effet menées pour la Chambre de la zakât par les membres des CP pour connaître les familles dans le besoin et le montant à assigner à celles qui sont imposables.

23. On gardera en tête, en plus des exemples mentionnés ici, les opérations réalisées grâce au sucre dans le quartier de Hajj Yûsuf.

24. Les quatre marchands évoqués sont engagés dans l'import-export, une activité qui requiert des fonds de roulement inaccessibles aux fortunes des particuliers sans l'appui des réseaux bancaires.

25. Nous dissocions le système politique de l'État pour n'en retenir qu'une définition minimum et circonstancielle, à savoir le système des conférences (nidhâm al-mu'tamarât). Guère différent d'un système parlementaire, il fonctionne sur la base d'assemblées populaires consultatives qui, 
sur une base pyramidale, élisent des organes exécutifs permanents ${ }^{\circ}$ les comités populaires, les conseils municipaux et d'État.

26. Un événement qui nous fut rapporté par un politicien local alors présent sur les lieux. Cet événement connut par ailleurs un certain écho dans la presse après que l'affaire eut été renvoyée devant la Justice. Ajoutons qu'après la découverte des 15 millions manquants, le président fut démis de ses fonctions.

27. Ces comités de marché, établis peu après la prise du pouvoir par le régime actuel, sont nommés par la municipalité et sont toujours partiellement composés de membres du système politique.

28. Il est bon de rappeler que nous avons affaire ici à une instance ${ }^{\circ}{ }^{\circ}$ civile ${ }^{\circ} »$ responsable du bon fonctionnement d'un marché. Revisité par le tandhîm shumûlîdu régime - une notion centrale dans le redéploiement de l'État au sein de la société civile après 1989 et qui peut se traduire approximativement par $^{\circ}$ système global ${ }^{\circ} »$ - le comité va notamment, via sa division « ${ }^{\circ}$ mobilisation politique ${ }^{\circ}$, remplir les voitures ou les bus des municipalités, afin de grossir les rangs des foules enthousiastes que l'on rencontre lors des cérémonies officielles.

29. Témoin, son application radicale en $1992 \mathrm{du}$ programme du FMI, pour laquelle, soit dit en passant, il ne devait recevoir aucune contrepartie.

30. Pour ne parler que des deux institutions redistributives au sein de l'État, il est à noter que la caisse de solidarité (Sundûq al-takâful al-ijtimâ'î) a fermé ses bureaux en juillet 1997 avec trois mois de retard dans le paiement de ses employés. Quant à la Chambre de la zakât, nonobstant la constante progression de ses revenus dès sa fondation en 1978, elle n'a pu mobiliser en 1996 que 1,35 milliard $€ s\left(888^{\circ} 000^{\circ} \$\right.$ ) (AWAD), pour un pays qui compte officiellement 19,2 millions de personnes au-dessous du seuil absolu de pauvreté. Ainsi, elle reste notoirement insuffisante aux dires mêmes des officiels. Pour des compléments d'information, voir Bakheet.

31. $~^{\circ}$ Who reaped most of the benefits, the beneficiaries being mostly near the center of power in Khartoum. The favourites consist mainly of top urban administrators and businessmen, although the priviledged persons tended to change with regimes. By contrast, rural administrators (tribal chiefs) and businessmen (pumpscheme owners and traders) have been in decline under most regimes. The result has been the redistribution of wealth in favour of urban elites ${ }^{\circ}$, Awad.

32. Le cas de Sudatel est instructif à ce propos $^{\circ}$ : la valeur initiale de la vente fut fixée à 250 millions \$. Cette vente eut lieu en privé, où seules furent conviées certaines personnalités. Le montant de cette dernière atteignit seulement 100 millions \$. Ensuite, durant les trois premiers mois de fonctionnement, un profit net de 5 millions $\$$ fut dégagé en augmentant considérablement les charges de service et en concentrant principalement les activités sur les relations avec l'étranger, aux dépens de la promotion des connections internes (Awad).

33. Les exemptions ont d'ailleurs atteint des proportions telles que cela a provoqué un conflit entre les marchands liés aux ONG et le ministre des Finances ${ }^{\circ}$; on estime en effet que le montant des exemptions représente plus de la moitié des revenus de l'imposition fiscale, laquelle se chiffre à 100 milliards £s, soit 66 millions \$ (entretien avec Awad).

34. On trouve notamment au rang des bénéficiaires de la zakât ${ }^{\circ}$ les mujâhidîn, les Forces de défense populaire, l'Organisation de l'appel au jihad, l'Organisation des martyrs, l'armée régulière.

35. Une expression populaire désignant l'extorsion ou la prédation des officiels.

36. Les associations de bienfaisance (jam'iyyât khayriyya) visant à assurer la logistique nécessaire aux cérémonies matrimoniales et funéraires - lesquelles relèvent de ce qu'on entend en général par la sphère du " $^{\circ}$ privé ${ }^{\circ} »$ - sont la dernière expression d'une vie associative indépendante. Tout le reste est tombé sous la supervision des $\mathrm{CP} .^{\circ}$

37. On se souvient du mariage de Hassan Tourabi avec la sœur de Sadek al-Mahdi, leader du parti Umma actuellement en exil au Caire.

38. Rafles de 1992-1993 : 400000 personnes déplacées dans les périphéries de la capitale. 
INDEX

Mots-clés : espace public, évergétisme, jihad, Soudan

\section{AUTEURS}

ISABELLE BAILLARD

Cedej

\section{PATRICK HAENNI}

Cedej 